\title{
Interventional Radiology Management of Renal Pseudoaneurysms: Experience at a Tertiary Care Hospital
}

\author{
Ujjwal Gorsi' ${ }^{\oplus} \quad$ Raghuraman Soundararajan $^{1} \quad$ Tejeshwar Singh Jugpal ${ }^{1 \oplus} \quad$ Anupam Lal $^{1}$ \\ Sreedhara B Shetty ${ }^{1} \quad$ Naveen Kalra ${ }^{1} \quad$ Mandeep Kang ${ }^{1} \quad$ Manavjit Singh Sandhu ${ }^{1}$
}

${ }^{1}$ Department of Radiodiagnosis, Post Graduate Institute of Medical

Address for correspondence Ujjwal Gorsi, MD, Department of Education and Research, Chandigarh, India Radiodiagnosis, Post Graduate Institute of Medical Education and Research (PGIMER), Chandigarh 160012, India (e-mail: ujjwalgorsi@gmail.com).

\begin{abstract}
Objectives Renal pseudoaneurysms are multifactorial in origin, and angioembolization remains the mainstay of treatment. Few case reports have also described percutaneous embolization using glue or thrombin. Our study aimed to evaluate the predictors of active bleed by analyzing their etiology, morphology, imaging features, and treatment adopted. To the best of our knowledge, this is the largest such study done in India. Methods This was a retrospective study of patients treated for renal pseudoaneurysms between 2014 and 2019. We reviewed their clinical data, treatment modalities used, and clinical outcomes. We also assessed computed tomography angiography (CTA) and conventional angiography images.

Results A total of 79 patients (54 males and 25 females) were included in the study. The mean age was 39.5 years (range $15-83$ years). The most common cause was renal biopsy, followed by surgery. Of these, three patients (3.9\%) had more than one lesion and $57 \%$ of pseudoaneurysms were seen in lower polar arteries. Active contrast extravasation was seen in $15.2 \%(n=12)$ of the patients and $21.5 \%(n=17)$ showed lobulations in CT and digital substraction angiography. The mean size of the pseudoaneurysms was $1.17 \mathrm{~cm}$ (SD 0.7); 22.8\% of pseudoaneurysms were wide necked. The embolization was approached by endovascular (89.9\%), percutaneous (10.1\%), or both (1.3\%) routes. Embolization was performed using microcoils (78.5\%), gel foam (12.7\%), N-butyl cyanoacrylate glue (8.9\%), polyvinyl alcohol (8.9\%), and thrombin $(5.1 \%)$ either as a single agent or in combination. The technical success was achieved in all cases after the first procedure. Pseudoaneurysms with a wide neck $(p=0.03)$ and lobulations ( $p=0.002$ ) were associated with active contrast extravasation. Episodes of

Keywords

- renal pseudoaneurysm

- angioembolization

- coils

- thrombin

- cyanoacrylate glue rebleeding were seen at a younger age ( $p$-value $=0.02$ ).

Conclusion Minimally invasive methods remain the cornerstone in the management of renal pseudoaneurysms with high success rates. The morphology of pseudoaneurysms can help predict the risk of active bleeding and decide the type of intervention. Direct percutaneous injection into the aneurysm sac is an alternate technique and should be considered when an endovascular approach is challenging.
\end{abstract}

\section{Introduction}

Renal pseudoaneurysm is described as an area of contained hematoma within the renal parenchyma or perinephric region, surrounded by adventitia of the vessel or renal parenchyma. ${ }^{1}$ These pseudoaneurysms usually show continuity with the arterial lumen. The differentiation of true from pseudoaneurysm is clinically relevant as pseudoaneurysms can lead to sudden massive hemorrhage. ${ }^{2}$ Renal pseudoaneurysms can occur after trauma or iatrogenic injuries following biopsy, percutaneous nephrostomy, and surgical nephrolithotomy. ${ }^{2}$ A pseudoaneurysm can be asymptomatic
License terms

(๑) (1) $\Theta \circledast$ 
or may present with hematuria, flank pain, and sudden fall in hematocrit. In a study, the incidence of perinephric hematoma after a renal biopsy was found to be $90 \%$, and only $6 \%$ were clinically significant. ${ }^{3}$ Therefore, early diagnosis and treatment are essential. Computed tomography angiography (CTA) has been the mainstay in the diagnosis of these pseudoaneurysms. ${ }^{1}$ Ultrasonography can be used for diagnosis, but it provides less information and may lead to false-negative results. No sufficient data are available in predicting the risk of hemorrhage in renal pseudoaneurysms.

The cornerstone of the treatment of pseudoaneurysm is selective endovascular angioembolization. ${ }^{4,5}$ Surgery is only reserved for patients with persistent bleeding despite minimally invasive management. Ultrasound-guided percutaneous injection of embolic agents into the pseudoaneurysm is done in patients who are hemodynamically stable and with unfavorable vascular anatomy or contrast allergy. ${ }^{6-8}$ Studies have shown success rates of $>90 \%$ with angioembolization. ${ }^{9}$ However, sufficient large study data are not available on the success rates of percutaneous embolization.

Our study aims to evaluate minimally invasive management of renal pseudoaneurysms with the intent to ascertain any imaging features associated with a high incidence of active bleeding.

\section{Materials and Methods}

We conducted a retrospective analysis in 79 patients of treated renal artery pseudoaneurysms from 2014 to 2019 in our department after approval from the institute's ethical committee. All patients underwent prior CTA for diagnosis and renal arterial anatomy mapping. All the procedures were done under local anesthesia with monitored anesthesia care. Written informed consent was obtained from all the patents. The demographic data, symptoms, hematological and hemodynamic parameters, treatment modalities used, and clinical outcomes were reviewed by two radiologists (-Table $\mathbf{1}$ ); CTA images and conventional angiography images were also reviewed. Characteristics like pseudoaneurysm size, neck, lobulations, location of pseudoaneurysm, and presence of active extravastion were analyzed.

The endovascular approach involves puncturing either the right or left common femoral artery using the modified Seldinger's technique. The arterial access is then secured by using a vascular access sheath. The choice of the diagnostic catheter for main renal artery cannulation in the present study was based on the morphology and course of renal arteries. Rosch Celiac 2 (RC2) catheter (most common), followed by Cobra 1 and Simmons 1 (all three catheters manufactured by Cook Medical) were used. After angiographic cannulation, runs were obtained to map out the renal and intrarenal arterial anatomy. The location of the pseudoaneurysm and the parent artery supplying the culprit lesion was identified. Selective and superselective cannulation of the affected artery was done using bi-axial system, with the microcatheter tip advanced as close to the aneurysm neck as possible. The number of pseudoaneurysms, site, size, and morphology were recorded. The choice of embolic agent was made based on the
Table 1 Demographic characteristics of the patients $(n=79)$

\begin{tabular}{|c|c|}
\hline Baseline characteristics & \\
\hline Age (years) & 39.9 \\
\hline Mean (range) & $16-83$ \\
\hline \multicolumn{2}{|l|}{ Sex } \\
\hline Male & 54 \\
\hline Female & 25 \\
\hline \multicolumn{2}{|l|}{ Hemoglobin at time of procedure $(\mathrm{g} / \mathrm{dL})$} \\
\hline$<5$ & 3 \\
\hline $5-10$ & 67 \\
\hline$>10$ & 9 \\
\hline \multicolumn{2}{|l|}{ Blood pressure $(\mathrm{mm} \mathrm{Hg})$} \\
\hline Systolic (mean) & $110 \mathrm{~mm} \mathrm{Hg}$ \\
\hline Diastolic (mean) & $76 \mathrm{~mm} \mathrm{Hg}$ \\
\hline \multicolumn{2}{|l|}{ Pulse (bpm) } \\
\hline Mean & 116 bpm \\
\hline \multicolumn{2}{|l|}{ Size of sac $(\mathrm{cm})$} \\
\hline Mean & $1.17 \mathrm{~cm}$ \\
\hline \multicolumn{2}{|l|}{ Site of pseudoaneurysm } \\
\hline Upper pole & 13 \\
\hline Mid pole & 20 \\
\hline Lower pole & 46 \\
\hline \multicolumn{2}{|l|}{ Neck of pseudoaneurysm } \\
\hline Narrow & 60 \\
\hline Wide & 19 \\
\hline \multicolumn{2}{|l|}{ Approach to embolization } \\
\hline Endovascular approach & 70 \\
\hline Percutaneous approach & 8 \\
\hline Both & 1 \\
\hline \multicolumn{2}{|l|}{ Agent in endovascular embolization } \\
\hline Coils only & 55 \\
\hline Coils with gelfoam slurry & 3 \\
\hline Coils with PVA particles & 2 \\
\hline Gelfoam only & 1 \\
\hline Glue only & 3 \\
\hline Coils with glue & 1 \\
\hline Glue with gelfoam & 1 \\
\hline Gelfoam with PVA & 3 \\
\hline \multicolumn{2}{|l|}{ Agents in percutaneous embolization } \\
\hline Commercially available thrombin & 4 \\
\hline Glue with lipiodol & 4 \\
\hline
\end{tabular}

Abbreviation: PVA, polyvinyl alcohol.

diameter of the parent artery, size of the neck of the pseudoaneurysm, accessibility by the endovascular route, and, needless to mention, the patient's affordability status. The most commonly used embolizing agents were microcoils. The dimension of the coils was decided based on the diameter and length of the stump of the parent feeding artery. Narrow neck aneurysms were defined as those with neck width $<4 \mathrm{~mm}$, or sac-to-neck ratio $>2$ ). Gelfoam and polyvinyl alcohol (PVA) particles were used in cases where sac was filling even after coil embolization of the feeding artery.) 
For the percutaneous access, the aneurysm sac was evaluated using gray-scale and Doppler sonography. The aneurysm sac appeared as a well-defined anechoic area within the renal parenchyma on the gray-scale with "ying-yang color flow" on Doppler images. The pseudoaneurysm sac was directly punctured percutaneously under sonography guidance using a long $22 \mathrm{G}$ spinal needle. We used commercially available thrombin (Tissel Lyo, Baxter Healthcare Ltd.) in these subset of patients. The vials contain lyophilized human fibrinogen (72-110 mg) and $500 \mathrm{IU}$ human thrombin per milliliter. The rate of thrombus formation was monitored using continuous sonography guidance. In seven patients, liquid embolizing agent N-butyl cyanoacrylate (NBCA) glue with lipoidal oil (1:2 or 1:1) was used. The glue injection was done under fluoroscopic guidance to look for adequate filling of the sac and prevent nontarget embolization. The patients were subsequently shifted from the angiography suite and were kept as an inpatient for monitoring and follow-up.

\section{Results}

We evaluated a total of 79 patients. The mean age was 39.5 years (range 15-83 years). The etiological factors included renal biopsy ( $n=36 ; 45.6 \%)$, surgery ( $n=32 ; 40.5 \%$ ), trauma $(n=5 ; 6.3 \%)$, percutaneous nephrostomy $(n=3 ; 3.8 \%)$, infection ( $n=2 ; 2.5 \%)$, and vasculitis ( $n=1 ; 1.3 \%)$. Of the 79 patients, three patients (3.9\%) had more than one lesion in different locations and fed by different branches. Around 57\% of the pseudoaneurysms were arising from lower polar arteries. Out of 79 patients, 8 had pseudoaneurysms associated with arteriovenous fistula. The mean size of the pseudoaneurysms was $1.17 \mathrm{~cm}$ (SD 0.7); 77.2\% showed narrow necks, and $22.8 \%$ of pseudoaneurysms were wide necked. Active contrast extravasation was seen in 12 patients (15.2\%). Seventeen patients showed pseudoaneurysm with lobulations in CT and DSA. In the pseudoaneurysms showing active extravasation, seven patients had lobulation and five had a smooth outline. Similarly, in the same patients with active contrast extravasation, seven had wide neck and five had narrow-necked pseudoaneurysms. The approach for embolization was endovascular $(89.9 \%)$, percutaneous $(10.1 \%)$, or both (1.3\%) routes. The agents used were coils in $78.5 \%(n=62)$ of cases ( - Fig. 1$)$. We used gel foam and PVA in $12.7 \%(n=10)$ and $8.9 \%(n=7)$ of cases, respectively ( - Fig. 1). Percutaneous embolization was done in eight cases. Of these, two patients had severe ostial stenosis of the renal artery on CTA, one patient had abdominal aortic aneurysm with aortoiliac stent graft in place, and the rest five had financial issues. We used NBCA glue and/or thrombin in four cases each ( $\mathbf{- F i g s .} \mathbf{2}$ and $\mathbf{3}$ ).

We achieved technical success in $100 \%$ of patients at the end of the first interventional procedure. The patients were followed-up for 48 hours post procedure. Clinical success after the primary intervention was $93.7 \%$. Five patients (7.6\%) had episodes of rebleeding, all approached
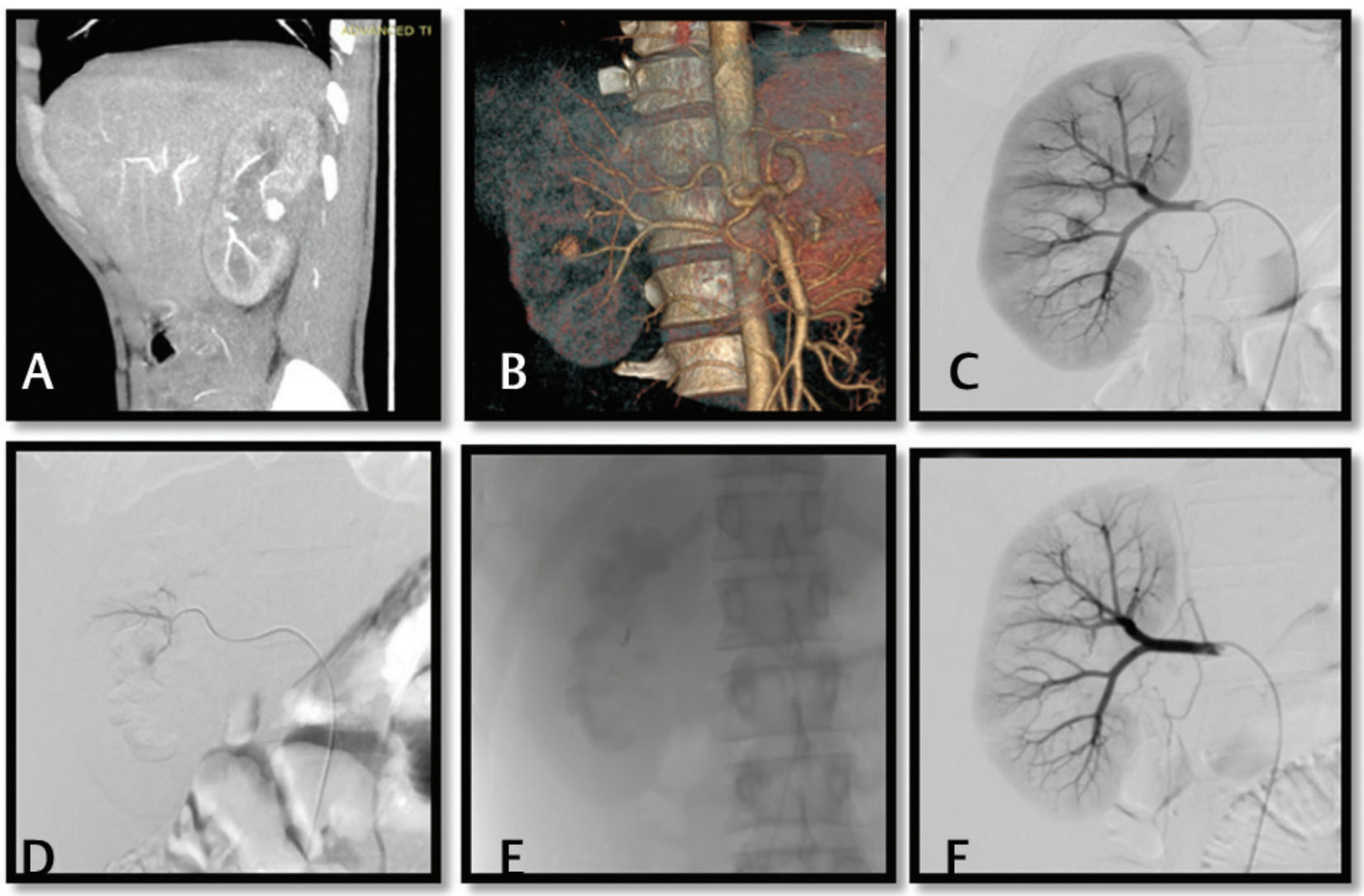

Fig. 1 Case of traumatic renal pseudoaneurysm. (A) Computed tomography angiogram showing a narrow neck pseudoaneurysm arising from one of the interpolar branches of the right renal artery. (B) Volume-rendered images showing the pseudoaneurysm. (C) Diagnostic DSA showing the pseudoaneurysm and the artery of origin. (D) Super selective run through microcatheter. (E) Coil placement under fluoroscopy guidance. (F) Post embolization run showing no filling of the sac. 


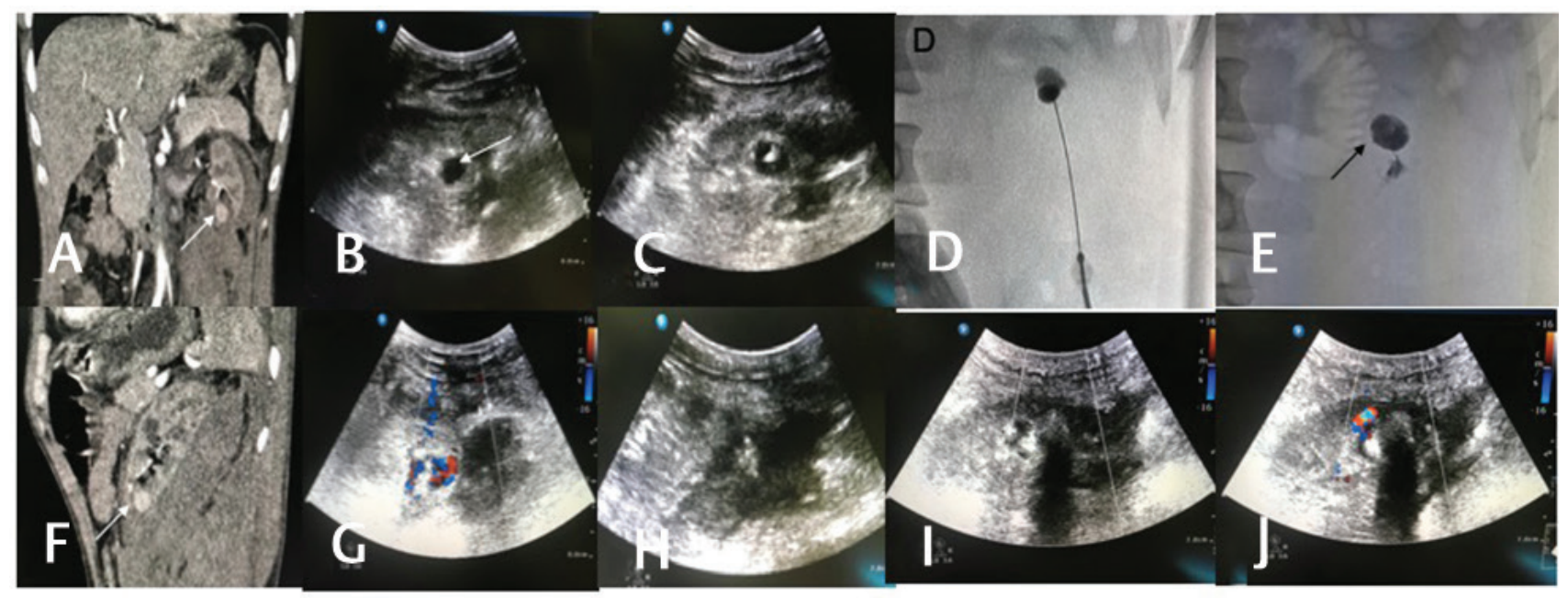

Fig. 2 Case of renal pseudoaneurysm following renal biopsy. (A,B) Computed tomography angiogram showing a narrow-necked pseudoaneurysm in the lower pole of left kidney (white arrow). (C) Gray-scale and color Doppler ultrasonography (USG) images showing the pseudoaneurysm (arrow). (D-F) USG-guided needle placement into the pseudoaneurysm and glue injection. (G,H) Fluoroscopic spots showing formation of glue casts (black arrow). (I,J) Postembolization USG-no filling of the pseudoaneurysm.

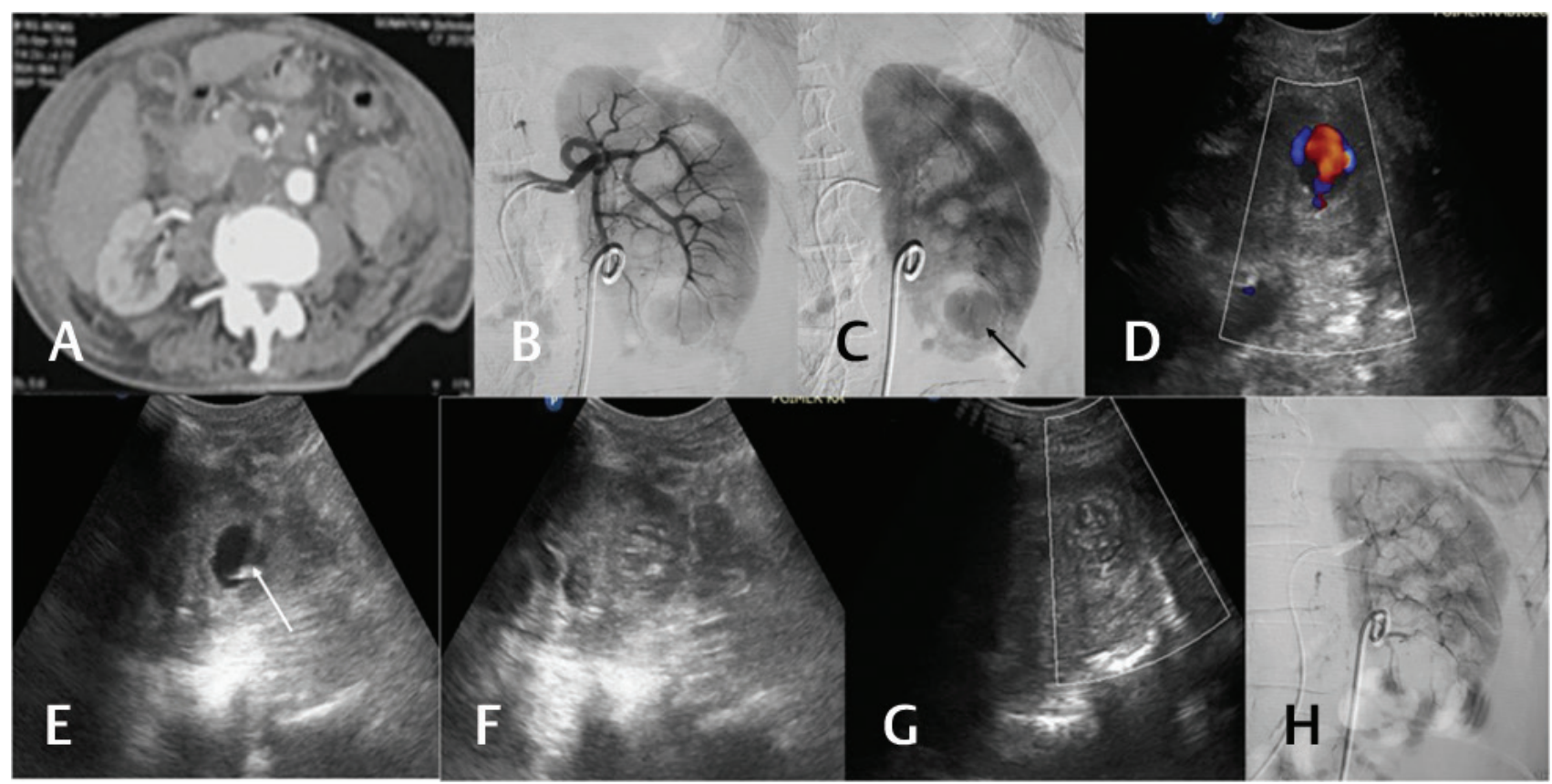

Fig. 3 (A) Left renal pseudoaneurysm post percutaneous nephrolithotomy. (B,C) DSA images showing the pseudoaneurysm in the lower pole (black arrow) with DJ stent in situ, the parent artery could not be selectively cannulated. (D) Color Doppler ultrasonography (USG) showing yin-yang pattern of color flow within the pseudoaneurysm. (E) Percutaneous placement of 22G spinal needle into the sac of pseudoaneurysm under USG guidance (white arrow). (F) Injection of thrombin into the pseudoaneurysm. (G) Color Doppler post thrombin embolization showing no color flow within the sac. (H) Post embolization DSA showing no opacification of the pseudoaneurysm.

with the percutaneous route. All five of these patients underwent subsequent endovascular embolization. No coagulopathy or other known reasons were observed in patients who had rebleeding. One patient who underwent endovascular coiling expired. We observed that pseudoaneurysms with a wide neck ( $p$-value $=0.03$ ) and lobulated outline ( $p$-value $=0.002$ ) were associated with active perinephric bleeding.

\section{Discussion}

Renal pseudoaneurysms can cause life-threatening hemorrhage in the perinephric space or renal collecting system. ${ }^{10}$ The risk of bleeding increases when the intravascular pressure exceeds the tamponade pressure provided by the containing tissues. Therefore, early diagnosis and treatment become necessary to prevent fatal complications. 
All cases of bleeding following renal biopsy or percutaneous procedures do not warrant invasive management unless there is presence of persistent hematuria or fall in patient's hematocrit. ${ }^{9}$ Minor degree of renal trauma such as contusions do not require urgent intervention. However, renal injury causing pseudoaneurysm or an arteriovenous fistula may require aggressive treatment. Earlier, surgical exploration was the only option available. However, with the advent of newer diagnostic techniques and catheter angiography, the need for surgery is reserved for patients with intractable bleeding despite angioembolization. CTA remains the primary modality for the diagnosis of renal pseudoaneurysm as it provides excellent spatial resolution with a lesser time of acquisition. ${ }^{1}$ It also provides additional information like active extravasation or complex morphology (i.e., multiple sites, lobulations, associated arteriovenous fistula). Ultrasonography can also be used in diagnosis; however, it has poor resolution, provides limited information, and has a high false-negative diagnostic rate. Catheter angiography is reserved for patients with diagnosed renal pseudoaneurysms as they provide an advantage of embolization in the same sitting, thus reducing radiation exposure.

Endovascular embolization is the modality of choice for treatment of renal pseudoaneurysms. The site of embolization should be close to the pseudoaneurysm neck to minimize renal parenchymal ischemia. ${ }^{11,12}$ Various embolic agents have been used, namely, platinum microcoils, cyanoacrylate glue, PVA particles, and gel foam. Percutaneous guided injection of thrombin or glue into sac is done when the pseudoaneurysm is inaccessible. ${ }^{13-15}$ The size of coils for endovascular embolization are decided based on the diameter of the feeding artery irrespective of the size of the pseudoaneurysm sac as the aim is to occlude the proximal parent artery harboring the aneurysm. The volume of the amount of embolizing agent used in percutaneous injection is decided according to the size of the sac of the pseudoaneurysm. Percutaneous embolization is not used in hemodynamically unstable patients. They are also contraindicated in cases where pseudoaneurysm is associated with arteriovenous fistula or communication with the pelvicalyceal system. To the best of our knowledge, our study is the largest series of renal pseudoaneurysms treated by an interventional radiology team in India. In our study, we aimed at analyzing renal pseudoaneurysms according to their imaging features and their minimal invasive management. The most common etiology in our subset of patients was secondary to percutaneous renal biopsy. Hence, lower pole was the most common site of pseudoaneurysm formation. We observed that pseudoaneurysms with wide neck and lobulated outlines had a higher incidence of active perinephric bleeding. In comparison, with previous literature, technical success was more in both endovascular and percutaneous embolization. However, the limitations in this study include short-term follow-up and small sample size.

\section{Conclusion}

Minimally invasive management remains the cornerstone in the management of renal pseudoaneurysms with good success rates and lesser complications.

\section{Funding}

None.

\section{Conflict of interest}

None.

\section{References}

1 Ngo TC, Lee JJ, Gonzalgo ML. Renal pseudoaneurysm: an overview. Nat Rev Urol 2010;7(11):619-625

2 Cura M, Elmerhi F, Bugnogne A, Palacios R, Suri R, Dalsaso T. Renal aneurysms and pseudoaneurysms. Clin Imaging 2011;35(1):29-41

3 Whittier WL, Korbet SM. Timing of complications in percutaneous renal biopsy. J Am Soc Nephrol 2004;15(1):142-147

4 Mohsen T, El-Assmy A, El-Diasty T. Long-term functional and morphological effects of transcatheter arterial embolization of traumatic renal vascular injury. BJU Int 2008;101(4):473-477

5 Pappas P, Constantinides C, Leonardou P, et al. Biopsyrelated hemorrhage of renal allografts treated by percutaneous superselective segmental renal artery embolization. Transplant Proc 2006;38(5):1375-1378

6 Siu YP, Tong MK, Leung KT, et al. Renal artery pseudoaneurysm following renal transplantation and treatment by percutaneous thrombin injection. Hong Kong Med J 2006;12(1):80-81

7 Gupta V, Galwa R, Khandelwal N, BapurajJR. Postpyelolithotomy renal artery pseudoaneurysm management with percutaneous thrombin injection: a case report. Cardiovasc Intervent Radiol 2008;31(2):422-426

8 Lal A, Singhal M, Ramachandran R, Rathi M, Jha V, Khandelwal N. Percutaneous injection of acrylic glue into renal allograft pseudoaneurysm for control of intractable post-biopsy hematuria. Indian J Nephrol 2014;24(2):124-126

9 Jain V, Ganpule A, Vyas J, et al. Management of non-neoplastic renal hemorrhage by transarterial embolization. Urology 2009;74(3):522-526

10 Yang HK, Koh ES, Shin SJ, Chung S. Incidental renal artery pseudoaneurysm after percutaneous native renal biopsy. BMJ Case Rep 2013;2013:bcr2012006537

11 Rivera M, Villacorta J, Jiménez-Alvaro S, Quereda C. Asymptomatic large extracapsular renal pseudoaneurysm following kidney transplant biopsy. Am J Kidney Dis 2011;57(1):175-178

12 Loffroy R, Guiu B, Lambert A, et al. Management of post-biopsy renal allograft arteriovenous fistulas with selective arterial embolization: immediate and long-term outcomes. Clin Radiol 2008;63(6):657-665

13 Krueger K, Zaehringer M, Strohe D, Stuetzer H, Boecker J, Lackner K. Postcatheterization pseudoaneurysm: results of US-guided percutaneous thrombin injection in 240 patients. Radiology 2005;236(3):1104-1110

14 Abud DG, Mounayer C, Benndorf G, Piotin M, Spelle L, Moret J. Intratumoral injection of cyanoacrylate glue in head and neck paragangliomas. AJNR Am J Neuroradiol 2004;25(9):1457-1462

15 GorsiU, Chaluvashetty S, Kalra N, et al. Percutaneous glue embolization as a primary treatment for visceral pseudoaneurysms. Minim Invasive Ther Allied Technol 2020;29(3):170-176 